\title{
VARIABILIDAD ESPACIAL DEL TRANSPORTE DE SEDIMENTO EN LA CUENCA SUPERIOR DEL RÍO ARAGÓN
}

\author{
A. NAVAS, B. VALERO-GARCÉS, L. GASPAR, J. M. GARCÍA-RUIZ, \\ S. BEGUERÍA, J. MACHÍN y M. LÓPEZ-VICENTE
}

\author{
Estación Experimental de Aula Dei-Instituto Pirenaico de Ecología
} Campus de Aula Dei, CSIC. Apartado 202, 50080 Zaragoza

Correo electrónico de contacto: anavas@eead.csic.es

RESUMEN. En el siglo pasado se produjo una importante regulación de los recursos hídricos en España con la construcción de numerosos embalses. En 1959, el río Aragón fue represado en Yesa (Depresión Interior del Pirineo Central) y desde entonces los sedimentos se han ido acumulando en su vaso, reduciendo así su capacidad inicial de almacenamiento de agua. En este trabajo se analiza el transporte de sedimentos en la red hidrológica del río Aragón desde la cabecera hasta el embalse de Yesa. Para ello se ha realizado el muestreo de aguas superficiales en 44 puntos distribuidos en las principales subcuencas del río Aragón. Se realizaron tres campañas de muestreo en invierno y dos en verano. Las concentraciones de sedimentos en suspensión, la conductividad eléctrica como una expresión del contenido en sólidos disueltos y el pH fueron analizados en las muestras de agua. Las mayores concentraciones de sedimento se registran en el muestreo de invierno en el río Esca (54.4 $\mathrm{mg} \mathrm{l}^{-1}$ ) y tras las tormentas del muestreo de verano en la cabecera del río Aragón (21.7 $\left.\mathrm{mg} \mathrm{l}^{-1}\right)$. El transporte de material disuelto es considerablemente inferior, entre 0.08 y $0.25 \mathrm{mg} \mathrm{l}^{-1}$. Las pautas de transporte de los materiales en suspensión se relacionan con factores como la litología, la cobertera vegetal y el uso del suelo en las subcuencas del río Aragón en Yesa. Los resultados de esta investigación son de interés para conocer los procesos de transporte de sedimentos en las cabeceras de cuenca de montaña, que son las principales fuentes de agua, e incrementar la información de utilidad para el manejo de embalses con el objetivo de reducir las tasas de acumulación de sedimentos.

ABSTRACT. The Aragón river was dammed at the foothills of the Pyrenean Inner Depression in 1959. Since then sediments are accumulated and its initial water storage capacity has been reduced. In this work, a study of the transport of sediments in the hydrological network of the Aragón river catchment until the 
Yesa reservoir has been undertaken based on the sampling of surface waters in 44 sites representative of the main subcatchments of the Aragón river. Three surveys were carried out in winter and summer. Total suspended sediments, electrical conductivity as an expression of dissolved solids content and $\mathrm{pH}$ were analysed in the water samples. Records of discharges at gauging stations of the water authorities were used as controls. The highest concentrations of suspended sediments occur during the winter sampling in the Esca river (54.4 $\left.\mathrm{mgl}^{-1}\right)$ and also after the storm events during the summer sampling in the headwaters of the Aragón river $\left(21.7 \mathrm{mg} \mathrm{l}^{-1}\right)$. The estimation of the dissolved solids indicates that the transport of materials in solution is much lower $\left(0.08-0.25 \mathrm{mg} \mathrm{l}^{-1}\right)$ than the suspended sediment load. The results provide information on the features of the transport of suspended and dissolved materials across the basin. The role played by lithology, vegetation cover, land use and drainage characteristics in the transport of sediments is examined for the different periods of sampling. Information gained with this research can be of interest as the headwater catchments in mountain areas are the main sources of water to lowlands. The knowledge on the process of sediment transport can be applied for the management of water bodies to prevent reservoir siltation.

Palabras clave: sedimentos en suspensión, CE, aguas superficiales, cabecera de cuenca, río Aragón, embalse de Yesa, Cuenca del Ebro, Pirineo central.

Key words: suspended sediment transport, EC, surface waters, catchment headwater, Aragón river, Yesa reservoir, Ebro basin, Central Spanish Pyrenees.

Enviado el 13 de Septiembre del 2007

Aceptado el 11 de abril de 2008

\section{Introducción}

Desde las primeras décadas del siglo XX se procedió a la regulación de ríos en cuencas españolas y a la construcción de embalses con objeto de incrementar las reservas de agua ante la incertidumbre y escasez de los recursos hídricos y por la creciente demanda de agua para los distintos usos. Actualmente, la colmatación de embalses es un importante problema medioambiental que afecta a numerosos embalses, reduciendo su capacidad de almacenamiento (Cobo et al., 1996).

A lo largo del siglo XXI se espera un aumento creciente de la demanda de agua por parte de una población cada vez más numerosa y una reducción de los recursos hídricos en amplias regiones del planeta entre las que se incluye la región mediterránea (Parry et al., 2000). La previsible reducción de los recursos hídricos se debe tanto a la expansión de la vegetación en relación con el abandono de tierras de cultivo en áreas de montaña como al aumento de temperaturas e incertidumbre de las precipitaciones como consecuencia del cambio climático (Easterling et al., 2000; Meehl et al., 2005). Las cabeceras de cuencas en zonas de montaña son las que más drásticamente 
pueden verse afectadas por procesos de cambio global (Machín et al., 2005). En este contexto cobra especial interés el análisis de la carga de sedimentos que son transportados por los ríos en diferentes periodos estacionales, correspondientes a aguas altas, bajas o en avenidas.

Valero et al. (1997) estudiaron las características sedimentológicas de los materiales depositados en el embalse de Barasona e indicaron que las mayores tasas de acumulación de sedimentos se habían producido en épocas de avenidas. Aunque no existe mucha información sobre los procesos de colmatación en embalses de cuencas de montaña, al igual que sucede en Barasona es probable que el suministro de sedimentos se relacione con periodos de deshielo así como con eventos de crecidas producidos tras fuertes tormentas (Navas et al., 2004).

Los efectos de la erosión, que en sectores de la cuenca del río Aragón es especialmente intensa (Navas et al., 1997, 2005a; Beguería, 2005), la consecuente generación de sedimentos y su posterior depósito en embalses afecta al desarrollo racional de los recursos de agua (Sundborg y Rapp, 1986). Como consecuencia del uso intensivo del suelo (Navas et al., 2005b), los problemas derivados del transporte y acumulación de sedimentos se agravan paulatinamente y requieren soluciones a corto plazo tanto en los lugares de erosión como en los de sedimentación.

Este estudio se centra en el análisis del transporte de los sedimentos en suspensión y de otros parámetros básicos de calidad de aguas (conductividad eléctrica y pH) en la cabecera de cuenca del río Aragón que vierte al embalse de Yesa. En este sector de cabecera existe gran diversidad de litologías, de vegetación y usos del suelo, así como variedad en las subcuencas, tanto morfométricas y de relieve como de densidad de drenaje, que son factores que se pretenden relacionar con la carga de sedimentos en los puntos de muestreo, para examinar así su importancia relativa. Los resultados de este estudio contribuirán a incrementar el conocimiento de los procesos de transporte de sedimentos en suspensión y del material disuelto, mediante la relación entre conductividad eléctrica y sólidos disueltos, en el sector de la cuenca del Aragón en Yesa. Ello servirá para establecer criterios sólidos para el manejo de embalses en un contexto cada vez más exigente de la conservación y sostenibilidad de los recursos.

\section{El área de estudio}

El área de estudio corresponde a la cuenca hidrográfica del río Aragón en el embalse de Yesa (Pirineo occidental español) y ocupa una superficie de $2191 \mathrm{~km}^{2}$ (Fig. 1). El Aragón es el río principal del área de estudio, además de varios afluentes por su derecha, en dirección N-S, que de este a oeste, son los ríos Lubierre, Estarrún, Aragón Subordán (con su afluente, el río Osía) el Veral y el Esca. Por su izquierda desembocan diversos ríos y barrancos procedentes de la cuenca molásica prepirenaica, de menor entidad. La organización de la red fluvial es probablemente pre-cuaternaria (Peña, 


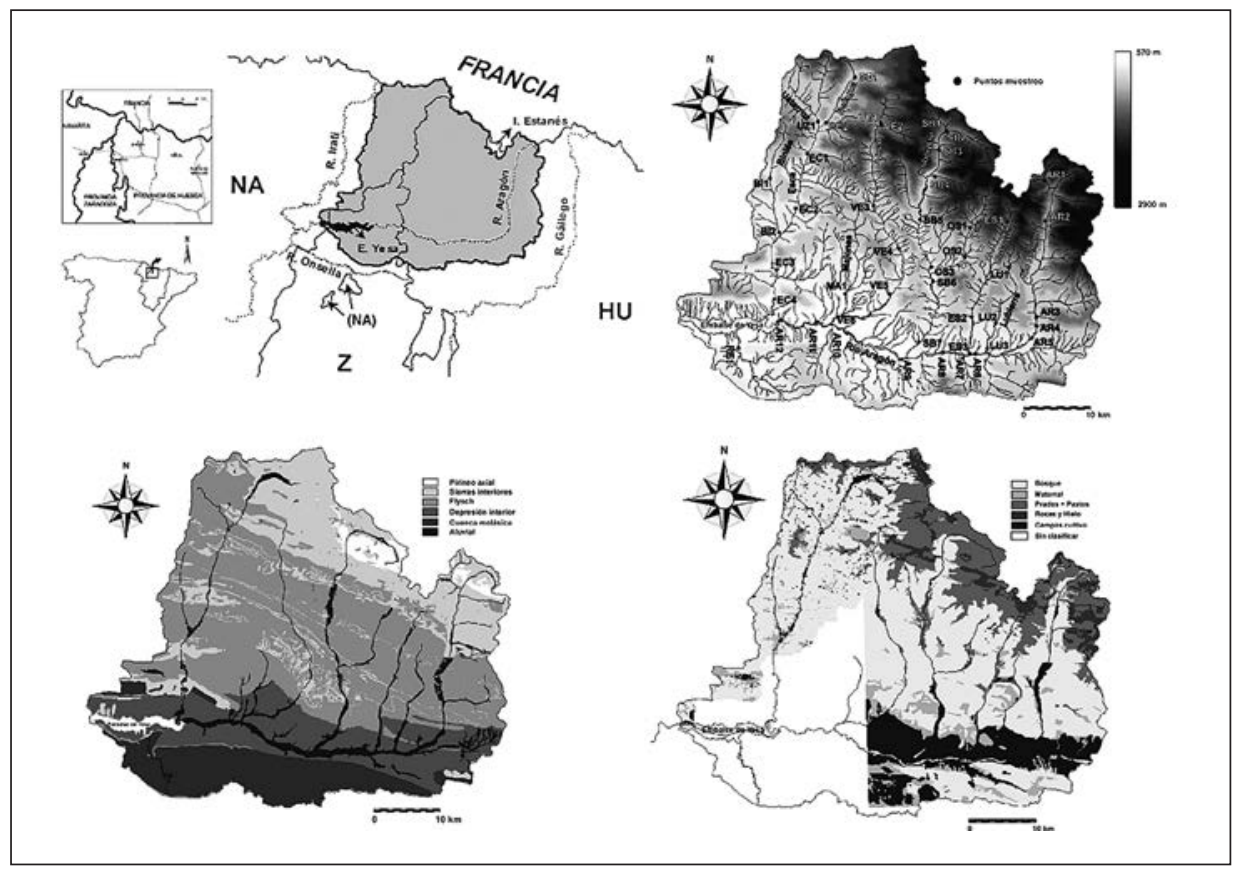

Figura 1. Situación geográfica del área de estudio, localización de los puntos de muestreo en los ríos de las subcuencas seleccionadas y a lo largo del cauce del Aragón, mapas de las principales unidades litológicas y de vegetación y usos del suelo

1991). En el Sector del Flysch, se aprecian niveles erosivos en las divisorias que sugieren la existencia temprana de jerarquización fluvial (Serrano, 1998).

El relieve presenta un gradiente general de sur a norte, con las principales alineaciones dispuestas en bandas de dirección WNW a ESE, fuertemente ligadas a la estructura geológica y la litología. La elevación media es de $1103.9 \mathrm{~m}$, y el gradiente altitudinal alcanza los $2406 \mathrm{~m}$ entre el punto más bajo, en la presa de Yesa (480 m) y el más alto, en el pico Collarada (2886 m).

En el área de estudio se distinguen varias unidades geoestructurales (Solé Sabarís, 1942; Soler y Puigdefábregas, 1970; Peña, 1991), que de norte a sur son el Pirineo Axial, las Sierras Interiores, el Sector del Flysch Eoceno, la Depresión Interior Altoaragonesa y la Cuenca molásica prepirenaica. Predomina el Sector del Flysch (52\% del total) y el resto de unidades ocupan superficies similares, en torno al 15\%, salvo el Pirineo Axial que está muy poco representado en el área de estudio (5\%). El Pirineo Axial constituye el núcleo de la cordillera Pirenaica. Las Sierras Interiores aparecen como una banda continua inmediatamente al sur del Pirineo Axial. Está integrada por rocas del Cretácico y el Paleoceno. El Sector del Flysch Eoceno consiste en una potente serie compuesta por capas alternantes de areniscas y lutitas margosas de espesor decimétrico. La Depresión Interior Altoaragonesa se sitúa sobre las margas del Eoceno medio, intensamente plegadas, sobre las que se instala un 
importante sistema de glacis y terrazas de edad cuaternaria. La Cuenca molásica prepirenaica está compuesta por lutitas, areniscas y conglomerados depositados durante el Eoceno medio y Oligoceno en facies deltaicas y de plataforma continental somera.

En la dinámica geomorfológica actual se pueden distinguir varios dominios en función de la altitud. El piso alpino está presente en el Pirineo Axial y las Sierras Interiores. El piso subalpino alpinizado aparece tanto en las dos unidades anteriores como en las partes más altas del Sector del Flysch. En él predominan los deslizamientos planares, incisiones paralelas y las cabeceras activas de barrancos. El piso montano se ve también afectado por frecuentes deslizamientos superficiales que suelen derivar en coladas de barro y derrubios (García-Ruíz y Puigdefábregas, 1982; Lorente et al., 2002). Por debajo de 1400 m, en especial en las laderas solanas, el fenómeno más espectacular es la formación de densas redes de cárcavas sobre las margas eocenas de la Depresión Interior. Una parte de los sedimentos movilizados en la cuenca llega hasta el embalse de Yesa.

El clima es de transición entre características del clima húmedo de tipo atlántico y rasgos mediterráneos continentalizados. La distribución de las temperaturas se relaciona con la altitud, mientras que la precipitación anual sigue un gradiente inverso, con los totales anuales de $800 \mathrm{~mm}$ en la Depresión Interior que aumentan hasta alrededor de $1000 \mathrm{~mm}$ en el Sector del Flysch y margen sur de las Sierras Interiores, y hasta más de $1500 \mathrm{~mm}$ al norte de las Sierras Interiores. El régimen de las precipitaciones acusa una influencia oceánica. (García Ruiz et al., 2001).

La distribución de la vegetación natural o potencial en el área de estudio está fuertemente condicionada por el relieve y las características climáticas y está profundamente alterada por las actividades humanas. Se distinguen los siguientes pisos de vegetación (Montserrat, 1971, 1988; Villar et al., 1997, 2001). El piso alpino aparece entre 2200-2400 y 2600-2800 m. Es el dominio de los pastizales de alta montaña. La vegetación clímax del piso subalpino, entre 1800 y 2000-2200 m, sería un pinar disperso de pino negro con matorrales diversos, cuya eliminación dio paso a pastizales. El piso altimontano y montano húmedo llega hasta 1650-1700 m (De la Riva, 1997) y está dominado por los pinares de Pinus sylvestris, los abetales y los hayedos. El sotobosque típico del pinar lo forman el boj (Buxus sempervirens) y el erizón (Echinospartum horridum). El piso mesomontano y supramediterráneo es el dominio de los robledales, sobre todo de Quercus pubescens. La transición hacia el supramediterráneo está marcada por encinares montanos de Quercus rotundifolia. El boj aparece en las etapas de degradación de ambos bosques. En el piso mesomediterráneo aparecen quejigares (Quercus faginea) y carrascales (Quercus rotundifolia) aunque la mayor parte de su área potencial se dedica a cultivos y pastoreo. Existen también diferentes formaciones azonales, como los bosques de ribera o las comunidades rupícolas.

\section{Métodos}

Los muestreos de agua en la red hidrológica del río Aragón hasta el embalse de Yesa se realizaron en tres campañas una en invierno (mes de enero) y dos en verano (mes 
de junio). Estas campañas pretenden ser representativas del contraste estacional de caudales. Una de las dos campañas de verano se realizó inmediatamente después de un episodio de lluvias generalizadas para analizar su efecto sobre el transporte de sedimentos en contraposición con la situación seca.

Se seleccionaron 44 puntos de muestreo (Fig. 1) que se localizan en las principales subcuencas de la cuenca del Aragón en Yesa. Las subcuencas de la margen izquierda que son de reducida extensión y gran homogeneidad litológica no se incluyeron en este estudio. En cada una de las subcuencas principales de la margen derecha se seleccionaron sitios de muestreo de cabecera a desembocadura representativos de los principales cambios litológicos, especialmente en el contacto entre las litologías carbonáticas de las Sierras Interiores, las areniscas y margas del Flysch y las facies de margas azules eocenas de la Depresión Interior. Las cuencas de menor longitud tienen menos puntos de muestreo: tres en los ríos Estarrún y Lubierre, nueve en el río Esca, siete en el río Veral, diez en el río Aragón Subordán y doce en el río Aragón, desde su cabecera a la desembocadura en el embalse de Yesa. En cada punto de muestreo se tomó un litro de agua en una botella de polietileno que se mantuvo refrigerada hasta su análisis en el laboratorio. Los parámetros analizados fueron sedimentos en suspensión tras filtrado y pesada, expresados en $\mathrm{mgl}^{-1}$, la conductividad eléctrica $\mathrm{dSm}^{-1}$, ya que su medida se relaciona con los sólidos disueltos a

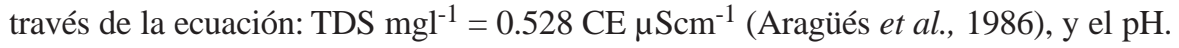

El análisis morfométrico de la cuenca del río Aragón en Yesa y de sus subcuencas se realizó para caracterizar mediante diferentes índices su forma y relieve. Se calcularon los siguientes índices: la relación de elongación (1), que relaciona el área (A) y la longitud de la cuenca ( $\mathrm{Lc}$ ), el índice de compacidad (2), que relaciona el perímetro ( $\mathrm{Pc}$ ) con el área, la circularidad (3), valor relacionado con el inverso de la compacidad, que permiten caracterizar la forma de la cuenca siendo los valores próximos a la unidad los que expresan una forma circular, la amplitud de relieve (Ah) o desnivel máximo, que se obtiene de la diferencia entre la cota máxima y mínima, y la relación de relieve (4).

$$
\begin{gathered}
\mathrm{Re}=\mathrm{A} /\left(\left(\pi \mathrm{Lc}^{2}\right) / 4\right) \\
\mathrm{Ic}=\mathrm{Pc} \pi /\left(2(\mathrm{~A} \pi)^{1 / 2}\right) \\
\mathrm{Cc}=4 \pi \mathrm{A} / \mathrm{Pc}^{2} \\
\mathrm{Rr}=\mathrm{Ah} / \mathrm{Lc}
\end{gathered}
$$

Se determinaron las características de la red de drenaje de la cuenca del río Aragón en Yesa mediante la estimación de la densidad de drenaje (5) que relaciona la longitud total de sus cauces con la superficie de la cuenca. Se elaboraron mapas de la red de drenaje de la cuenca y subcuencas de la zona de estudio y se calcularon la relación de bifurcación (Rb), que es la relación del número de cauces de un orden cualquiera entre el número de cauces de orden inmediatamente superior, y el coeficiente de torrencialidad, que expresa el número de cauces de orden 1 por $\mathrm{Km}^{2}$ y se relaciona con la capacidad de erosión de una cuenca.

$$
\mathrm{D}=\mathrm{L} / \mathrm{S}
$$


Para analizar el efecto de la litología, vegetación y usos del suelo sobre el transporte de sedimentos se utilizaron los mapas de base de vegetación del ITGME a escala 1:50000, hojas $n^{\circ} 117$ (Ochagavia), 118 (Zuriza), 143 (Navascues), 144 (Ansó) 145 (Sigües) 176 (Jaca) y 177 (Biescas) obtenidos a partir de la cartografía 1:50000 del proyecto de estudio del medio físico y de sus riesgos naturales en un sector del Pirineo Central (IGTME y DGA) elaborado por el Instituto Pirenaico de Ecología, además del mapa de vegetación proporcionado por el servicio web de la Diputación Foral de Navarra para su territorio y el litológico a escala 1:100000 que se integraron en un SIG. En ambos casos dado el nivel de detalle se realizó un trabajo de síntesis para agrupar y unificar litologías y vegetación en un total de 6 clases. Las clases litológicas diferenciadas se asocian con las principales unidades geoestructurales. Las clases establecidas para la vegetación fueron bosque, matorral, prados y pastos, roca desnuda, cultivos y miscelánea.

\section{Características de las subcuencas del Aragón en Yesa}

Los resultados de la caracterización morfométrica y de relieve de las subcuencas estudiadas se presentan en la Tabla 1. En la Tabla 2 se recogen las características de la red de drenaje, incluyendo los datos referentes a la longitud del cauce principal, del total

Tabla 1. Índices morfométricos de la forma y el relieve de las cuencas y subcuencas del río Aragón

\begin{tabular}{|l|r|r|c|c|c|c|c|c|c|c|}
\hline & $\mathbf{1}$ & $\mathbf{2}$ & $\mathbf{3}$ & $\mathbf{4}$ & $\mathbf{5}$ & $\mathbf{6}$ & $\mathbf{7}$ & $\mathbf{8}$ & $\mathbf{9}$ & $\mathbf{1 0}$ \\
\hline ESCA & 522.6 & 257.6 & 0.54 & 3.18 & 0.10 & 68.6 & 990 & 2900 & 500 & 2.86 \\
Belagua & 89.1 & 53.0 & 0.50 & 1.58 & 0.40 & 106.7 & 1320 & 2200 & 600 & 6.67 \\
Biniés & 50.8 & 45.2 & 0.17 & 1.79 & 0.31 & 41.0 & 900 & 1500 & 700 & 5.13 \\
Ustárroz & 70.4 & 37.6 & 0.93 & 1.27 & 0.62 & 132.6 & 1110 & 1900 & 600 & 10.2 \\
\hline VERAL & 201.9 & 109.4 & 0.16 & 2.17 & 0.21 & 42.5 & 1100 & 2300 & 600 & 2.5 \\
Majones & 71.6 & 19.0 & 0.25 & 1.56 & 0.41 & 52.6 & 900 & 1500 & 500 & 5.26 \\
\hline SUBORDÁN & 357.5 & 108.2 & 1.79 & 1.61 & 0.38 & 41.6 & 1320 & 2700 & 600 & 1.98 \\
Osía & 76.4 & 46.3 & 0.22 & 1.49 & 0.45 & 95.2 & 1280 & 2700 & 700 & 4.76 \\
\hline ESTARRÚN & 84.4 & 65.3 & 0.20 & 2.01 & 0.25 & 86.2 & 1070 & 2700 & 700 & 1.53 \\
\hline LUBIERRE & 46.9 & 40.8 & 0.18 & 1.68 & 0.35 & 76.5 & 1060 & 2100 & 700 & 5.46 \\
\hline ARAGÓN & 2192.2 & 257.6 & 0.34 & 1.55 & 0.42 & 26.7 & 1025 & 2900 & 500 & 1.14 \\
\hline
\end{tabular}

1: Area $\left(\mathrm{km}^{2}\right)$; 2: Perímetro $(\mathrm{Km})$; 3: Relación de enlogación; 4: Índice de compacidad; 5: Circularidad; 6 : Relación de relieve; 7: altitud media (m); 8: Cota máxima (m); 9: Cota mínima (m); 10: Pendiente media (\%). 
de cauces, la densidad de drenaje, relaciones de bifurcación entre los ríos de distintos órdenes y el coeficiente de torrencialidad. Los índices morfométricos indican que todas las cuencas son alargadas. La cuenca del Esca $\left(523 \mathrm{Km}^{2}\right)$, que comprende las subcuencas de los ríos Ustárroz, Belagua y Biniés, presenta un bajo número de cauces de orden 1 y las relaciones de bifurcación indican que la evolución entre cauces de orden 1, 2 y 3 está en conjunto más desarrollada que en otras cuencas. La cuenca del Veral con $202 \mathrm{Km}^{2}$ (excluyendo a la del Majones) es la más alargada y su red de drenaje está bien desarrollada siendo su coeficiente de torrencialidad de los más altos. La cuenca del Aragón Subordán presenta el desnivel más alto de todas las cuencas estudiadas. En conjunto la red de drenaje está más desarrollada que en las otras cuencas y presenta valores intermedios tanto de densidad de drenaje como de índice de torrencialidad. La subcuenca del río Osía, afluente por la izquierda del río Aragón Subordán, presenta una forma muy alargada y una red de drenaje mal desarrollada. La cuenca del río Estarrún $\left(84 \mathrm{Km}^{2}\right)$ tiene una red de drenaje formada por un gran número de cauces de orden 1 que unido a la fuerte pendiente dan un funcionamiento torrencial al río. La cuenca del río Lubierre es la de mayor pendiente; su red de drenaje está muy poco desarrollada y su coeficiente de torrencialidad es bajo.

Tabla 2. Índices de drenaje de las subcuencas y cuenca del río Aragón

\begin{tabular}{|c|c|c|c|c|c|c|c|c|}
\hline & \multirow{2}{*}{$\begin{array}{c}\text { long. } \\
\text { cauce } \\
\text { principal } \\
\text { Km }\end{array}$} & \multirow{2}{*}{$\begin{array}{c}\text { long. } \\
\text { total } \\
\text { cauces } \\
\text { Km }\end{array}$} & \multirow{2}{*}{$\begin{array}{c}\text { densidad } \\
\text { drenaje } \\
\mathrm{Km} / \\
\mathrm{Km}^{2}\end{array}$} & \multicolumn{4}{|c|}{ Relación de bifurcación } & \multirow{2}{*}{$\begin{array}{c}\text { Coef. } \\
\text { torrencialidad }\end{array}$} \\
\hline & & & & $1^{\circ} / 2^{\circ}$ & $2^{\circ} / 3^{\circ}$ & $3^{\circ} / 4^{\circ}$ & $4^{\circ} / 5^{\circ}$ & \\
\hline ESCA & 35.0 & 439.2 & 0.84 & 3.1 & 5.0 & 8.0 & & 0.24 \\
\hline Belagua & 9.8 & 28.2 & 0.32 & 3.8 & 2.0 & & & 0.33 \\
\hline Biniés & 15.0 & 38.6 & 0.76 & 2.5 & & & & 0.09 \\
\hline Ustárroz & 19.5 & 64.3 & 0.91 & 3.3 & 3.0 & & & 0.20 \\
\hline VERAL & 40.0 & 363.1 & 1.80 & 4.1 & 20.0 & & & 0.41 \\
\hline Majones & 19.0 & 112.6 & 1.57 & 3.1 & 3.3 & 3.0 & & 0.43 \\
\hline SUBORDÁN & 50.5 & 371.8 & 1.04 & 4.2 & 5.8 & 5.0 & & 0.34 \\
\hline Osía & 21.0 & 91.3 & 1.19 & 3.5 & 10.0 & & & 0.46 \\
\hline ESTARRÚN & 23.2 & 97.5 & 1.15 & 5.1 & 7.0 & & & 0.43 \\
\hline LUBIERRE & 18.3 & 43.7 & 0.93 & 5.5 & & & & 0.23 \\
\hline ARAGÓN & 88.0 & 1988.1 & 0.91 & 3.4 & 6.7 & 3.7 & 7.0 & 0.27 \\
\hline
\end{tabular}


La distribución porcentual de las principales clases litológicas y de vegetación y usos del suelo en las subcuencas se presenta en las Tablas 3 y 4, respectivamente. Dado que las subcuencas tienen una distribución N-S, las litologías que afloran siguen la ordenación de su afloramiento desde la zona axial hasta las margas eocenas. En cabecera de las cuencas del Esca y Aragón Subordán, y en menor medida del río Veral, afloran grauwacas, pizarras en facies turbidíticas y rocas efusivas básicas de la zona axial y calizas masivas de las Sierras Interiores, si bien en todas ellas predominan los materiales del Flysch, y hacia las zonas de desembocadura aparecen las margas eocenas de la depresión media. Las cuencas de los ríos Estarrún y Lubierre se asientan casi en exclusiva sobre los materiales del Flysch y sólo al llegar a la desembocadura afloran las margas eocenas. En cuanto a la vegetación, domina el bosque sobre todas las litologías, las praderas y pastos en la zona axial y los campos de cultivo sobre los aluviales. Una distribución similar se observa en la cuenca del Veral si bien los prados de alta montaña adquieren en esta cuenca mayor importancia.

Tabla 3. Distribución porcentual de las principales litologías en las unidades geoestructurales en la cuenca superior del río Aragón y en las subcuencas estudiadas

\begin{tabular}{|l|c|c|c|c|c|}
\hline & $\begin{array}{c}\text { calizas masivas } \\
\text { calizas y margas }\end{array}$ & $\begin{array}{c}\text { margas y } \\
\text { areniscas }\end{array}$ & margas & lutitas & aluvial \\
\hline & $\begin{array}{c}\text { Sierras } \\
\text { Interiores }\end{array}$ & Flysch & $\begin{array}{c}\text { Depresión } \\
\text { Interior }\end{array}$ & $\begin{array}{c}\text { Cuenca } \\
\text { molásica }\end{array}$ & \\
\hline $\begin{array}{c}\text { Paleozoico- } \\
\text { Mesozoico }\end{array}$ & Eoceno & Eoceno & $\begin{array}{c}\text { Eoceno- } \\
\text { Oligoceno }\end{array}$ & Cuaternario \\
\hline ESCA & 29.6 & 64.3 & 2.2 & 1.7 & 2.3 \\
Belagua & 78.9 & 15.3 & 0 & 0.5 & 5.3 \\
Biniés & 16.7 & 82.4 & 0 & 0 & 0.9 \\
Ustárroz & 11.5 & 88.2 & 0 & 0 & 0.3 \\
\hline VERAL & 42.1 & 49.2 & 6.9 & 0.1 & 1.7 \\
Majones & 8.7 & 68.1 & 19.7 & 0 & 3.5 \\
\hline SUBORDÁ́N & 27.4 & 56.5 & 2.1 & 9.6 & 4.5 \\
Osía & 19.8 & 77.6 & 0 & 0 & 2.6 \\
\hline ESTARRÚN & 15.4 & 73.5 & 5.1 & 0 & 6.0 \\
\hline LUBIERRE & 1.7 & 72.3 & 17.8 & 0 & 8.2 \\
\hline ARAGÓN & 33.1 & 39.0 & 14.6 & 5.5 & 7.9 \\
\hline
\end{tabular}


Tabla 4. Distribución porcentual de la vegetación y los usos del suelo en la cuenca del río Aragón y en las subcuencas estudiadas

\begin{tabular}{|l|c|c|c|c|c|c|}
\hline & $\begin{array}{c}\text { bosque } \\
\%\end{array}$ & $\begin{array}{c}\text { matorral } \\
\text { \% }\end{array}$ & $\begin{array}{c}\text { prados y } \\
\text { pastos } \\
\text { \% }\end{array}$ & $\begin{array}{c}\text { roca y } \\
\text { hielo } \\
\text { \% }\end{array}$ & $\begin{array}{c}\text { campos } \\
\text { de cultivos } \\
\%\end{array}$ & $\begin{array}{c}\text { sin } \\
\text { clasificar } \\
\text { \% }\end{array}$ \\
\hline ESCA & 60.9 & 6.2 & 8.6 & 2.9 & 2.3 & 19.0 \\
Belagua & 59.0 & 2.1 & 20.1 & 14.5 & 4.3 & 0 \\
Biniés & 84.0 & 6.8 & 4.4 & 0 & 2.5 & 2.3 \\
Ustárroz & 74.5 & 1.4 & 20.3 & 2.8 & 1.1 & 0 \\
\hline VERAL & 61.5 & 5.4 & 23.6 & 2.7 & 3.5 & 3.3 \\
Majones & 23.8 & 5.4 & 0 & 0 & 1.3 & 69.5 \\
\hline SUBORDÁN & 53.0 & 5.1 & 32.3 & 5.7 & 3.9 & 0 \\
Osía & 72.0 & 0.5 & 23.5 & 3.6 & 0.4 & 0 \\
\hline ESTARRÚN & 55.4 & 10.1 & 19.5 & 7.1 & 7.8 & 0 \\
\hline LUBIERRE & 54.4 & 16.3 & 6.3 & 0 & 23.0 & 0 \\
\hline ARAGÓN & 43.6 & 6.7 & 13.5 & 3.4 & 10.0 & 22.8 \\
\hline
\end{tabular}

\section{Transporte de sedimentos en suspensión}

La mayor concentración de sedimentos en suspensión se registra en las subcuencas occidentales, con valores que alcanzan los $54.4 \mathrm{mgl}^{-1}$ en la cabecera del río Esca. De las tres campañas realizadas es en el muestreo de invierno donde más sedimentos se transportan (rango desde inapreciable a $54.4 \mathrm{mgl}^{-1}$ ), mientras que en el muestreo de verano se registran concentraciones inferiores que están comprendidas entre 0.2 y $6.2 \mathrm{mgl}^{-1}$. En el muestreo tras las tormentas, las cuencas del sector occidental (Esca y Veral) presentan concentraciones hasta un 50\% más bajas que en el de verano, con mínimos de 0.65 y 0.77 $\mathrm{mgl}^{-1}$ y máximos de 2.7 y $1.7 \mathrm{mgl}^{-1}$ en los ríos Esca y Veral respectivamente. Una disminución porcentual similar se registra también en las cuencas del Lubierre y Estarrún, con mínimos de 0.76 y $0.62 \mathrm{mgl}^{-1}$ y máximos de 1.1 y $3.9 \mathrm{mgl}^{-1}$ respectivamente. En contraposición, en el Aragón Subordán se duplican las concentraciones tras las tormentas con respecto a las del muestreo de verano alcanzando los $4.8 \mathrm{mgl}^{-1}$. Esta variación en las concentraciones de sedimentos tras las tormentas en las distintas subcuencas podría deberse a que el muestreo se inició en las cuencas orientales por lo que el pico de crecida podría haber pasado cuando se muestrearon las cuencas occidentales.

Como se observa en la Fig. 2a, la concentración de sedimentos en el Esca es hasta 10 veces mayor en el muestreo de invierno (mínimo: $0.5 \mathrm{mgl}^{-1}$ en el muestreo de vera- 
no, máximo: $54.4 \mathrm{mgl}^{-1}$ en el de invierno). En la cuenca del Veral las concentraciones son similares y también de rango muy variado entre el muestreo de verano e invierno con un mínimo de $0.5 \mathrm{mgl}^{-1}$ y un máximo de $51.2 \mathrm{mgl}^{-1}$. En el Aragón Subordán varía entre 0.2 y $44.3 \mathrm{mgl}^{-1}$, máximo que se registra en el muestreo de invierno dentro del sector del Flysch, siguiendo la misma pauta del Esca y Veral.

El comportamiento de las cuencas más orientales es diferente a las anteriores (Fig. 2b). En estas cuencas la concentración de sedimentos en suspensión es mucho menor, los máximos son hasta 10 veces más bajos y además las mayores concentraciones se registran en el muestreo de verano. En el Estarrún el rango de concentración es de 0.5 a $5.8 \mathrm{mgl}^{-1}$ y en el Lubierre de 0.7 a $6.2 \mathrm{mgl}^{-1}$.

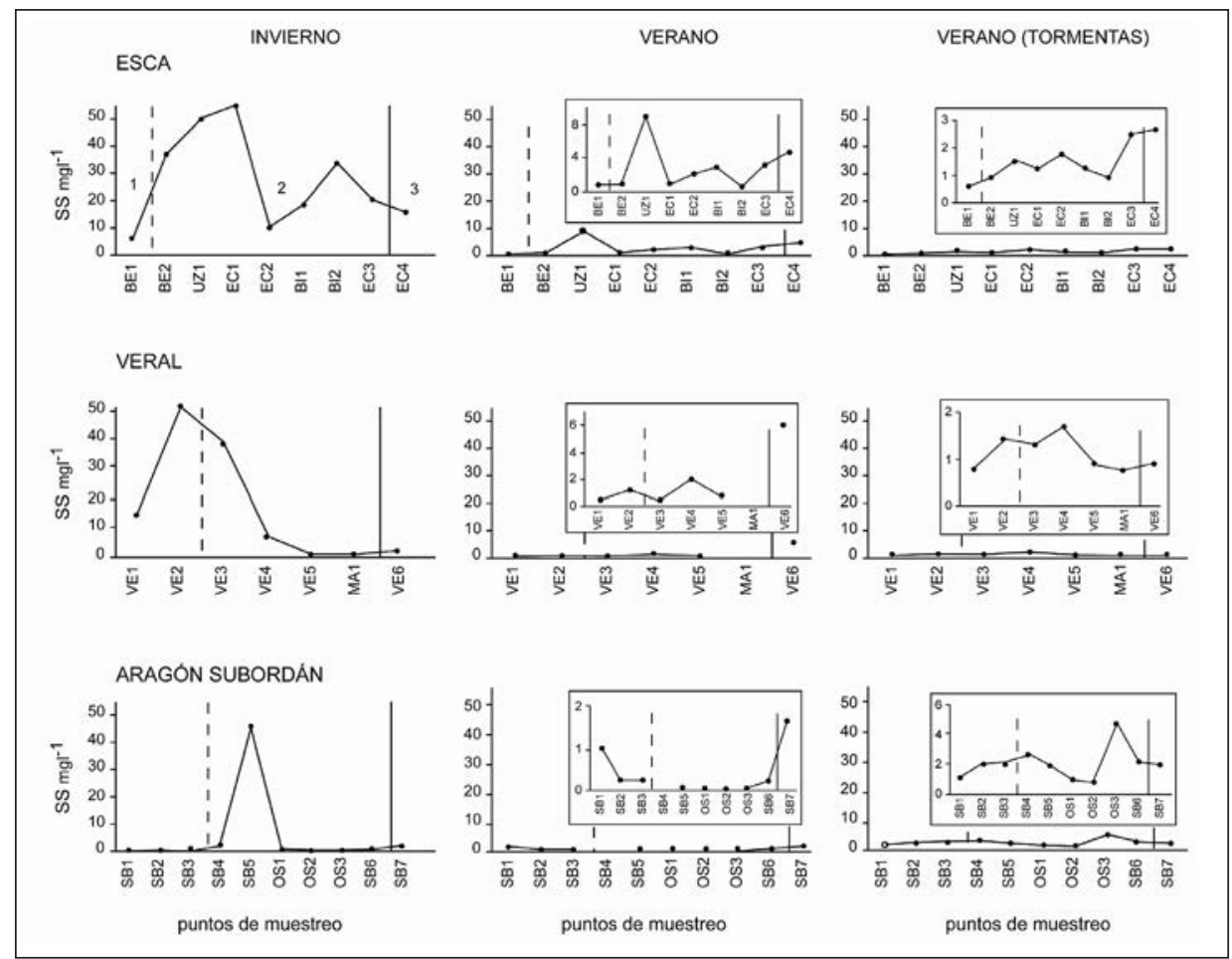

Figura 2. a) Concentraciones de sedimento en suspensión en las tres campañas de muestreo en las subcuencas de los ríos Esca, Veral y Aragón Subordán. Las líneas verticales representan los límites entre las litologías de 1) las Sierras Interiores, 2) el Flysch, 3) las margas eocenas de la Depresión Interior

El comportamiento de las tres cuencas más occidentales (Esca, Veral y Subordán) es similar. Registran su máxima concentración en el muestreo de invierno, y de la cabecera a la desembocadura los sedimentos se incrementan pasando de valores muy bajos 
en la zona de las Sierras Interiores a aumentar considerablemente en el Flysch, con excepción del punto VE2 en el Veral. En el muestreo de verano no se registran oscilaciones tan bruscas siendo la máxima concentración de $9 \mathrm{mgl}^{-1}$ en el Esca y de 6 y $1.6 \mathrm{mgl}^{-1}$ en el Veral y Subordán, respectivamente. En el muestreo tras las tormentas la concentración de sedimentos disminuye y el máximo es de tan solo $2.7 \mathrm{mgl}^{-1}$.

Las cuencas orientales del Estarrún y Lubierre (Fig. 2b) muestran una pauta claramente diferente, con máximos de concentración en el muestreo de verano y mínimos en el de invierno y tras las tormentas. En general, la concentración de sedimentos es mayor una vez atravesados los materiales del Flysch, lo que indicaría una mayor contribución de las margas eocenas al aporte de sedimentos, si bien las diferencias no son muy acusadas.

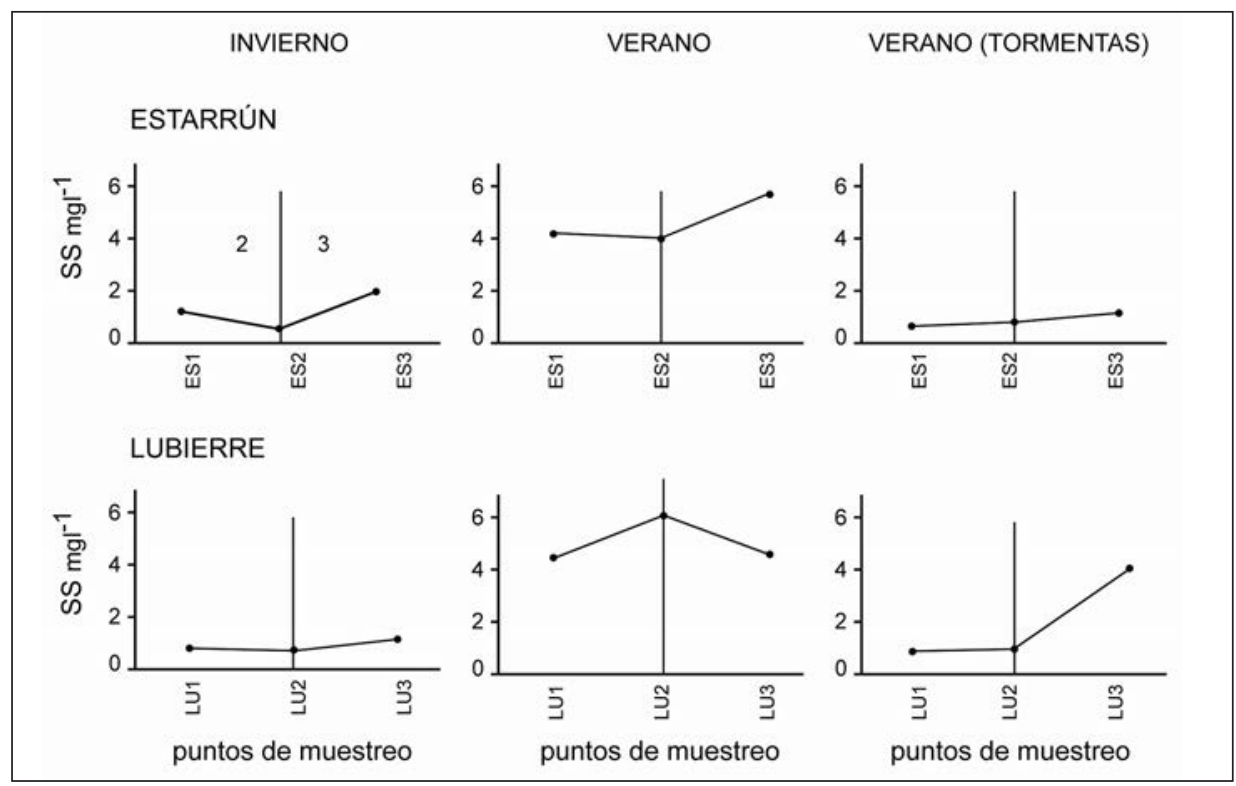

Figura 2. b) Concentraciones de sedimento en suspensión en las tres campañas de muestreo en las subcuencas de los ríos Estarrún y Lubierre. La línea vertical representa el límite entre las litologías de 2) el Flysch y 3) las margas eocenas de la Depresión Interior

\section{Conductividad eléctrica, sólidos disueltos y pH}

Los valores de CE son bajos, entre 0.152 y $0.480 \mathrm{dSm}^{-1}$, aunque dentro de este rango las diferencias entre valores de muestreo de invierno y verano llegan a ser del 50\%. Como se observa en la Fig. 3, la cuenca del Esca presenta la CE mas alta en el muestreo de invierno $\left(0.480 \mathrm{dSm}^{-1}\right)$ que es también el que tiene mayor carga de sedimentos, y la mínima de $0.232 \mathrm{dSm}^{-1}$ tras las tormentas. En la cuenca del Veral la pauta es similar y los valores máximo y mínimo son de 0.459 y $0.218 \mathrm{dSm}^{-1}$, respectivamente. La cuenca del Aragón Subordán registra en el muestreo de invierno tanto su máximo $\left(0.386 \mathrm{dSm}^{-1}\right)$, 


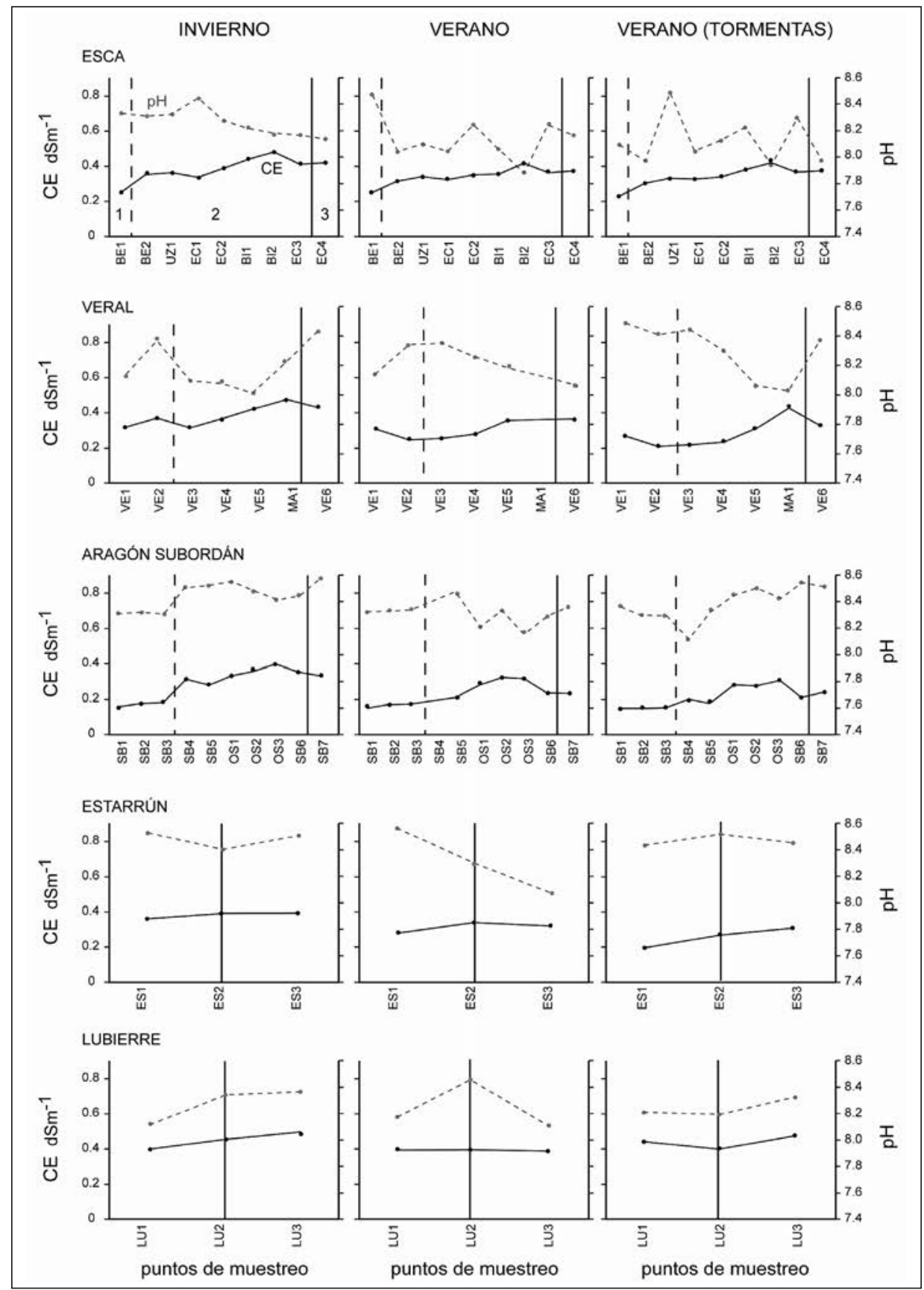

Figura 3. Valores de conductividad eléctrica y de pH en las tres campañas de muestreo en las subcuencas del río Aragón. Las líneas verticales representan los límites entre las litologías de 1) las Sierras Interiores, 2) el Flysch, 3) las margas eocenas de la Depresión Interior 
como su mínimo $\left(0.152 \mathrm{dSm}^{-1}\right)$, que es el valor más bajo de las cuencas estudiadas. También en el Estarrún el máximo se da en invierno $\left(0.368 \mathrm{dSm}^{-1}\right)$ y el mínimo $(0.174$ $\mathrm{dSm}^{-1}$ ) se registra en el muestreo tras las tormentas. En la cuenca del Lubierre no hay gran diferencia entre valores, que oscilan entre 0.378 y $0.471 \mathrm{dSm}^{-1}$, que es el máximo y corresponde al muestreo de invierno.

La conductividad eléctrica es ligeramente más alta en los puntos de muestreo ubicados en el sector más meridional del Flysch para las cuencas occidentales mientras que en las cuencas más orientales, en las que el cambio litológico es sólo del Flysch a las margas este ligero ascenso se marca al entrar en contacto con las margas eocenas. En general, la conductividad eléctrica tiende a aumentar desde cabecera hasta la desembocadura en las subcuencas estudiadas.

En cuanto a los sólidos disueltos estimados a partir de su relación lineal con la CE $(\mathrm{r}=0.598)$ para el río Aragón en Yesa, y según se observa en la Tabla 5, sus concentraciones son notablemente inferiores a las de los sedimentos en suspensión. Los valores mayores, que son muy similares en todas las subcuencas y varían entre 0.19 y $0.25 \mathrm{mgl}^{-1}$ en el Subordán y el Esca, respectivamente, se registran en el muestreo de invierno y en

Tabla 5. Concentración de los sólidos disueltos (SD $\left.\mathrm{mgl}^{-1}\right)$ en las subcuencas y cuenca del río Aragón en los tres muestreos, calculados a partir de la relación de la conductividad eléctrica

\begin{tabular}{|l|c|c|c|c|}
\hline & Invierno & Verano & $\begin{array}{c}\text { Verano } \\
\text { (Tormentas) }\end{array}$ \\
\hline \multirow{6}{*}{ Esca } & BE1 & 0.129 & 0.129 & 0.122 \\
& BE2 & 0.188 & 0.164 & 0.161 \\
& UZ1 & 0.190 & 0.177 & 0.175 \\
& EC1 & 0.177 & 0.168 & 0.173 \\
& EC2 & 0.206 & 0.183 & 0.181 \\
& BI1 & 0.231 & 0.185 & 0.203 \\
& BI2 & 0.253 & 0.215 & 0.220 \\
& EC3 & 0.215 & 0.190 & 0.196 \\
& EC4 & 0.220 & 0.195 & 0.199 \\
\hline \multirow{6}{*}{ Veral } & VE1 & 0.162 & 0.155 & 0.140 \\
& VE2 & 0.188 & 0.126 & 0.115 \\
& VE3 & 0.161 & 0.127 & 0.115 \\
& VE4 & 0.184 & 0.140 & 0.126 \\
& VE5 & 0.213 & 0.178 & 0.165 \\
& MA1 & 0.242 & 0.000 & 0.229 \\
& VE6 & 0.221 & 0.182 & 0.174 \\
\hline
\end{tabular}


Tabla 5 (continuación). Concentración de los sólidos disueltos ( $\left.S D \mathrm{mgl}^{-1}\right)$ en las subcuencas y cuenca del río Aragón en los tres muestreos, calculados a partir de la relación de la conductividad eléctrica

\begin{tabular}{|c|c|c|c|c|}
\hline & & Invierno & Verano & $\begin{array}{c}\text { Verano } \\
\text { (Tormentas) }\end{array}$ \\
\hline \multirow[t]{10}{*}{ Aragón Subordán } & SB1 & 0.080 & 0.092 & 0.082 \\
\hline & SB2 & 0.089 & 0.096 & 0.083 \\
\hline & SB3 & 0.093 & 0.098 & 0.088 \\
\hline & SB4 & 0.157 & 0.000 & 0.108 \\
\hline & SB5 & 0.142 & 0.115 & 0.101 \\
\hline & OS1 & 0.167 & 0.161 & 0.154 \\
\hline & OS2 & 0.184 & 0.178 & 0.148 \\
\hline & OS3 & 0.204 & 0.174 & 0.165 \\
\hline & SB6 & 0.178 & 0.131 & 0.113 \\
\hline & SB7 & 0.169 & 0.131 & 0.131 \\
\hline \multirow[t]{3}{*}{ Estarrún } & ES1 & 0.177 & 0.143 & 0.092 \\
\hline & $\mathrm{ES} 2$ & 0.192 & 0.172 & 0.136 \\
\hline & ES3 & 0.194 & 0.163 & 0.153 \\
\hline \multirow[t]{3}{*}{ Lubierre } & LU1 & 0.201 & 0.205 & 0.231 \\
\hline & LU2 & 0.233 & 0.202 & 0.211 \\
\hline & LU3 & 0.249 & 0.200 & 0.211 \\
\hline \multirow[t]{10}{*}{ Aragón } & AR1 & 0.093 & 0.070 & 0.059 \\
\hline & AR2 & 0.137 & 0.099 & 0.073 \\
\hline & AR3 & 0.208 & 0.147 & 0.130 \\
\hline & AR4 & 0.244 & 0.146 & 0.130 \\
\hline & AR5 & 0.157 & 0.103 & 0.092 \\
\hline & AR7 & 0.214 & 0.218 & 0.249 \\
\hline & AR8 & & 0.147 & 0.243 \\
\hline & AR10 & 0.195 & 0.143 & 0.135 \\
\hline & AR11 & 0.220 & 0.150 & 0.140 \\
\hline & AR12 & 0.206 & 0.153 & 0.139 \\
\hline
\end{tabular}

la parte baja de las subcuencas. En contraposición, las menores concentraciones (entre $0.08 \mathrm{mgl}^{-1}$ en el Aragón Subordán y $0.12 \mathrm{mgl}^{-1}$ en el Esca) se producen en la zona de cabecera de las subcuencas en el muestreo tras las tormentas. 
El pH de las aguas en la red de drenaje del Aragón es alcalino, su rango de valores es muy homogéneo y las diferencias no llegan a un orden de magnitud siendo el mínimo de 7.9 en el Esca y el máximo de 8.6 en el Estarrún (Fig. 3). Estos valores son muy similares en los distintos muestreos y para todas las cuencas estudiadas, de forma que el pH no presenta una pauta diferencial de cabecera a desembocadura en los ríos estudiados y su escaso rango de variación no se asocia claramente a cambios litológicos, de vegetación o usos del suelo.

\section{Variaciones de los sedimentos en suspensión, CE, sólidos disueltos y pH a lo largo del cauce del Aragón}

Desde la cabecera hasta la desembocadura del río Aragón en el embalse de Yesa se distribuyen un total de doce puntos de muestreo (Fig. 1), siendo los cinco primeros de zona de cabecera propiamente, en el AR6 se incorpora el río Lubierre, en el AR7 el río Estarrún, en el AR9 el río Aragón Subordán y en el AR11 el río Veral.

La mayor concentración de sedimentos en suspensión se produce en el muestreo tras las tormentas (Fig. 4), de manera que es en la cabecera del río donde se alcanza el máximo de $21.7 \mathrm{mgl}^{-1}$ para luego disminuir notablemente hasta valores de entre 4 y 1.2 $\mathrm{mgl}^{-1}$ en la zona de presa. Valero et al. (1999) y Navas et al. (2004) asocian altas tasas de sedimentación en Barasona con avenidas del Ésera en un registro sedimentario de más de 70 años en el vaso del embalse. La mayor concentración de sedimentos en el río Aragón es casi un 60 \% inferior al máximo de invierno registrado en el Esca, lo que se relaciona con el mayor caudal que el río Aragón registró tras las tormentas. En contraposición, la mayor concentración en el río Esca en Sigüés que se produjo en el muestreo de invierno coincide con los más altos caudales como se observa en los datos de aforos de la CHE correspondientes a las campañas de muestreo (Tabla 6).

En el muestreo de invierno se registra la más baja concentración de sedimento en suspensión, que varía entre 0.07 y $5.5 \mathrm{mgl}^{-1}$. Los bajos valores de cabecera podrían interpretarse, al igual que se observó en la cuenca del Ésera (Navas et al., 1998), como resultado de menores tasas de erosión, propias de un paisaje cubierto por la nieve durante el invierno.

En el muestreo de verano las concentraciones de sedimento son también bajas, entre 0.4 a $10.3 \mathrm{mgl}^{-1}$. Estas bajas concentraciones tanto en el muestreo de invierno como de verano se relacionan con la disminución del transporte de partículas por la menor escorrentía, como se desprende de los valores de los caudales (Tabla 6). Asimismo, es en el muestreo de verano cuando se registra un paulatino incremento de los sedimentos a lo largo del cauce hasta el embalse de Yesa.

Para el conjunto de la cuenca del río Aragón en Yesa se han establecido correlaciones entre las concentraciones de sedimento en suspensión en los distintos muestreos y la superficie ocupada por los distintos usos del suelo, los porcentajes de las principales lito- 


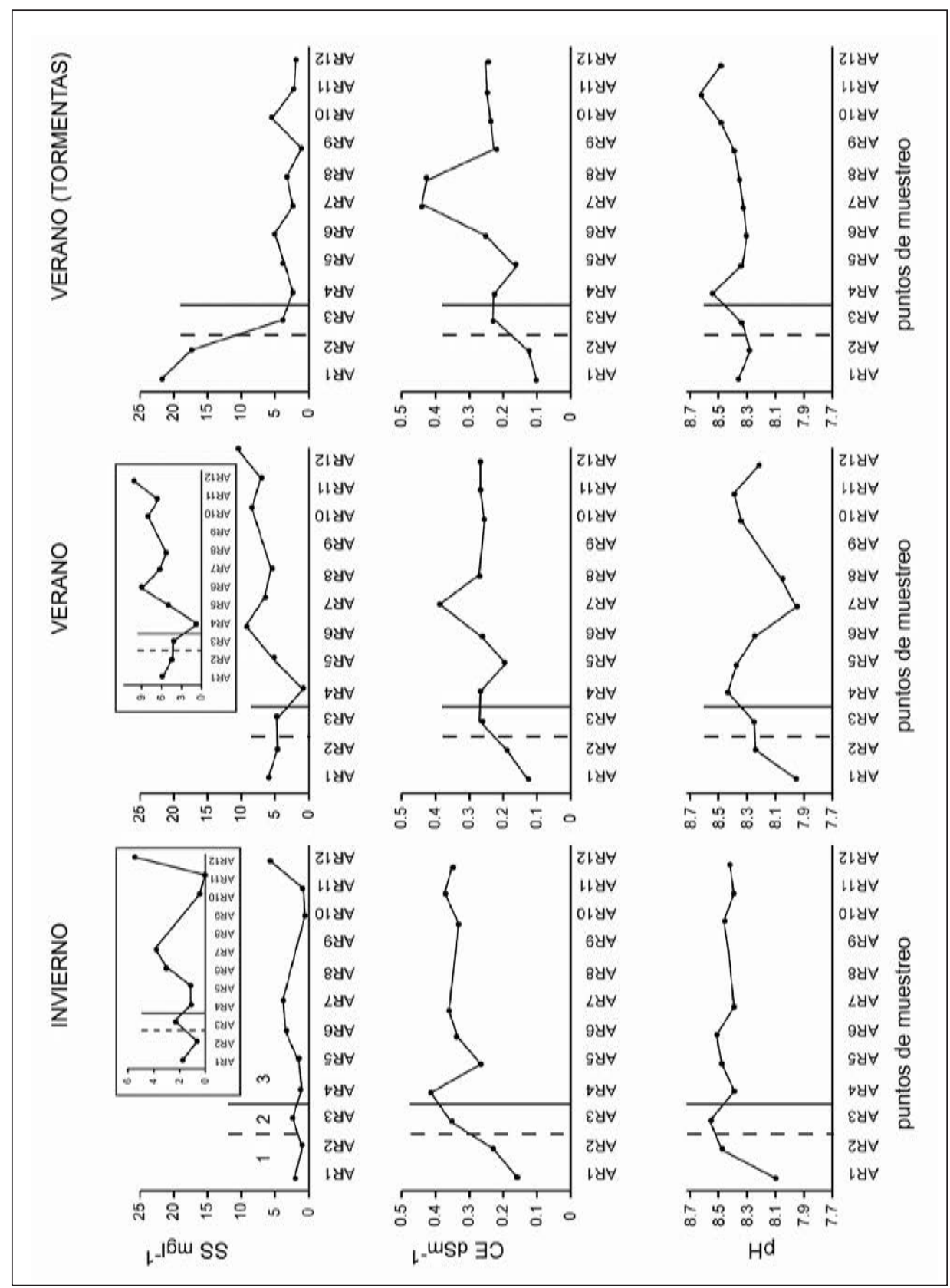

Figura 4. Concentraciones de sedimento en suspensión y valores de conductividad eléctrica y $\mathrm{pH}$ en las tres campañas de muestreo en la cuenca del río Aragón. Las líneas verticales representan los límites entre las litologías de 1) las Sierras Interiores, 2) el Flysch, 3) las margas eocenas de la Depresión Interior 
Tabla 6. Caudales $\left(Q \mathrm{~m}^{3} \mathrm{~s}^{-1}\right)$ en las estaciones de aforo de la CHE en la cuenca superior del río Aragón correspondientes a los días de los muestreos de invierno (I), verano (V) $y$ tras las tormentas $(T)$

\begin{tabular}{|l|c|c|c|c|}
\hline & $\begin{array}{c}\text { Aragón- } \\
\text { Canfranc } \\
\text { A271 }\end{array}$ & $\begin{array}{c}\text { Aragón-Jaca } \\
\text { A018 }\end{array}$ & $\begin{array}{c}\text { Javierregay } \\
\text { A061 }\end{array}$ & $\begin{array}{c}\text { Aragón cola- } \\
\text { Yesa } \\
\text { A170 }\end{array}$ \\
\hline muestreo & $\mathbf{Q}$ & $\mathbf{Q}$ & $\mathbf{Q}$ & $\mathbf{Q}$ \\
\hline I & 0.88 & 5.8 & 11.9 & 23.8 \\
V & 0.94 & 0.58 & 5.96 & 15.65 \\
T & 4.51 & 7.34 & 16.21 & 45.08 \\
\hline & Esca-Isaba & $\begin{array}{c}\text { Esca-Sigüés } \\
\text { A063 }\end{array}$ & $\begin{array}{c}\text { Veral-Biniés } \\
\text { A170 }\end{array}$ \\
\hline muestreo & $\mathbf{A 2 6 8}$ & $\mathbf{Q}$ & $\mathbf{Q}$ & \\
\hline I & $\mathbf{Q}$ & 14.45 & 4.93 & \\
V & 3.53 & 2.17 & 1.27 & \\
T & 1.12 & 4.11 & 3.97 & \\
\hline
\end{tabular}

logías y la densidad de drenaje. Como se observa en la Tabla 7, la relación con la densidad de drenaje en las distintas subcuencas no es significativa en ninguno de los muestreos. Las mayores concentraciones de sedimento en suspensión, especialmente en el muestreo de invierno, se relacionan directamente con la extensión de las superficies de cultivos, pastos y prados en las subcuencas, lo que demuestra el efecto del cultivo del suelo sobre la generación de sedimento. Este hecho coincide con lo descrito por Valero et al. (1999), que encuentran un mayor aporte de sedimentos en la cuenca del Ésera coincidiendo con el sector de la misma en el que los cultivos son más extensos. En el muestreo tras las tormentas las correlaciones no son significativas, lo que probablemente se debe a la mayor variabilidad de la ocurrencia de escorrentía, que enmascararía el efecto de los otros factores de cubierta y uso del suelo. En cuanto al factor litológico, existe una clara relación entre las concentraciones de sedimento y la erodibilidad de los materiales, ya que los valores de los coeficientes son mas altos en las margas eocenas, tanto en el muestreo de invierno como en verano. Sin embargo esto no sucede en el muestreo tras las tormentas que podría también explicarse por la variabilidad espacial de la escorrentía y su efecto preponderante sobre los otros factores.

Los valores más bajos de conductividad eléctrica (Fig. 4) se registran en la cabecera de la cuenca siendo muy similares en los tres muestreos (invierno: $0.176 \mathrm{dSm}^{-1}$, verano: $0.132 \mathrm{dSm}^{-1}$, tras las tormentas: $0.111 \mathrm{dSm}^{-1}$ ). Se observa una tendencia a su ascenso desde cabecera hasta la zona del Flysch, mientras que en las margas eocenas se pro- 
Tabla 7. Coeficientes de correlación entre las concentraciones de sedimentos en suspensión y la superficie ocupada por los distintos usos del suelo, los porcentajes de las principales litologías y la densidad de drenaje en las tres campañas de muestreo en la cuenca superior del río Aragón

\begin{tabular}{|l|c|c|c|}
\hline & Invierno & Verano & Verano (tormentas) \\
\hline bosque & 0.748 & 0.536 & 0.261 \\
bosque y matorral & 0.771 & 0.547 & 0.263 \\
cultivos & 0.926 & 0.587 & 0.276 \\
Pastos y prados & 0.779 & 0.486 & 0.349 \\
cultivos, pastos y prados & 0.861 & 0.545 & 0.349 \\
\hline calizas masivas y margas & 0.101 & 0.224 & 0.487 \\
flysch & 0.254 & 0.016 & 0.488 \\
margas & 0.678 & 0.517 & 0.121 \\
\hline densidad de drenaje & 0.083 & 0.009 & 0.102 \\
\hline
\end{tabular}

duce un descenso que se interrumpe con la incorporación de las aguas del Lubierre, de modo que la CE aumenta y llega a alcanzar en este punto los valores más altos registrados a lo largo del cauce con 0.413 y $0.472 \mathrm{dSm}^{-1}$, respectivamente, en los muestreos de verano y tras las tormentas, para luego descender aguas abajo hacia la desembocadura.

En cuanto a los sólidos disueltos estimados a partir de la CE (Tabla 5), sus rangos varían entre 0.06 y $0.25 \mathrm{mgl}^{-1}$, que es el máximo tras las tormentas. Como cabe esperar por la distribución de las litologías, los valores más bajos se encuentran en la zona de cabecera y paulatinamente van ascendiendo hasta alcanzar las máximas concentraciones en el punto donde se incorporan las aguas del Lubierre. En general, es en el muestreo de invierno donde se alcanzan las mayores concentraciones, lo que reflejaría los diferentes procesos de incorporación a las aguas de las partículas disueltas y en suspensión.

A lo largo del curso del río Aragón, no se observan variaciones importantes en el pH (Fig. 4), siendo el valor mínimo 7.96 en el muestreo de verano y el máximo de 8.64 tras las tormentas, que es el muestreo en el que el pH presenta, en general, unas pautas más homogéneas en los puntos de muestreo. No obstante su distribución no se puede asociar con las de la litología, la vegetación y usos del suelo.

\section{Conclusiones}

Este trabajo ha utilizado tres campañas de muestreo de agua en 44 puntos de la cuenca superior del río Aragón, con el fin de observar irregularidades espaciales y relacionar- 
las con diferentes factores ambientales así como con actividades humanas. A pesar de las limitaciones del procedimiento (por ejemplo, la toma de muestras no se produjo en el mismo momento en todo la cuenca debido al tamaño del muestreo), los resultados reflejan la influencia tanto de la litología como de la cubierta vegetal y los usos del suelo en la producción de sedimento. Las mayores concentraciones de sedimento se transportan al embalse de Yesa coincidiendo con caudales altos, ya sea de invierno como en el caso del Esca o tras las tormentas como en la cabecera del Aragón. Este hecho subraya la importancia del régimen hídrico de los ríos, que ha de tenerse en cuenta a la hora de elaborar posibles estrategias de prevención de aterramiento de los embalses. El transporte de materiales disueltos es significativamente inferior y muestra diferentes pautas de aportes.

La heterogeneidad de la cuenca en cuanto al suministro de sedimentos debido a diferentes litologías, vegetación y usos del suelo evidencia la variabilidad de los procesos sedimentarios en las cuencas mediterráneas de montaña. Un mejor conocimiento de los procesos de erosión, transporte y sedimentación es necesario para promover un manejo más adecuado de un recurso limitado como el agua.

Los valores mas altos de sedimento en suspensión se registran en áreas con los mayores porcentajes de tierras cultivadas. La correlación entre valores de sedimento en suspensión y los usos del suelo indica que el impacto antropogénico ha de considerarse como un factor significativo en la caracterización de subáreas geográficas respecto a su capacidad de emisión de sedimentos.

Las cabeceras de cuenca constituyen las principales áreas suministradoras de agua. Por ello, el análisis de las relaciones entre el transporte de sedimento, por un lado, y los usos del suelo, vegetación y litología, por otro, proporciona criterios científicos de base para atenuar la colmatación de embalses y optimizar la gestión de los recursos hídricos imprescindible en un contexto de necesaria sostenibilidad de los recursos de suelo y agua

\section{Agradecimientos}

Esta investigación ha sido financiada por los proyectos CICYT, REN2003-09130C02-02/CLI, CGL2005-02009/BTE y CGL2006-11619/HID. Confederación Hidrográfica del Ebro ha facilitado las series de datos de aforos del río Aragón y el resumen de datos histórico.

\section{Referencias bibliográficas}

Aragües, R., Alberto, F. y Quilez, D. (1986). El Sistema Integrado del Ebro. Relaciones entre los parámetros químicos de las aguas superficiales de la cuenca del Ebro. 728 pp. ISBN: 84-398-7293-3. 
BEGUERÍA, S. (2005). Identificación y caracterización de las fuentes de sedimento en áreas de montaña: Erosión y transferencia de sedimento en la cuenca alta del río Aragón. IPE-CSIC, 158 pp., Zaragoza.

Cobo, R., Gómez, J. L., Plata, A., Sanz, M. E. y Avendaño, C. (1996). Influencia del régimen de explotación del embalse de Puentes en las tasas de sedimentación durante el periodo 1954-1994. Actas V Jornadas Españolas de Presas: 155-169. Valencia

De LA RIVA, J. (1997). Los montes de la Jacetania. Caracterización física y explotación forestal. Consejo de Protección de la Naturaleza de Aragón, (Serie Investigación $\mathrm{n}^{\circ}$ 10), 358 pp., Zaragoza

Easterling, D.R., Meehl, G.A., Parmesan, C., Changnon, S.A., Karl, T.R. Y MEARns, L.O. (2000). Climate Extremes: Observations, Modeling and Impacts. Science, 289: 2068-2074.

García-Ruiz, J.M. y Puigdefábregas, J. (1982). Formas de erosión en el Flysch Eoceno surpirenaico. Cuadernos de Investigación Geográfica, 8: 85-128.

García-Ruiz, J.M., Beguería, S., LóPez Moreno, J.I., Lorente, A. y Seeger, M. (2001). Los recursos hídricos superficiales del Pirineo aragonés y su evolución reciente. Geoforma ediciones, 192 pp., Logroño.

LOREnTE, A., GARCíA-RuIZ, J.M., BEgueríA, S. y ARNÁEZ, J.M. (2002). Factors explaining the spatial distribution of hillslope debris flows. A case study in the Flysch Sector of the Central Spanish Pyrenees. Mountain Research and Development 22 (1): 32-39.

Machín, J., NaVAs, A., Domenech, S. y LóPeZ-Vicente, M. (2005). El rió Arlanza en cabecera: evolución reciente y tendencias en condiciones de cambio global. Cuadernos de Investigación Geográfica, 31: 77-95.

Meehl, G.A., Washington, W.M., Collins, W.D., Arblaster, J.M., Hu, A., Buja, L.E., STRAND, W.G. Y TENG, H. (2005). How much more global warming and sea level rise. Science, 307: 1769-1772.

Monserrat, P. (1971). La Jacetania y su vida vegetal, Publicaciones de la Caja de Ahorros de Zaragoza, Aragón y Rioja, 108 pp., Zaragoza

Monserrat, P. (1988). Flora. Enciclopedia temática de Aragón, Vol. 6, Ediciones Moncayo, 323 pp., Zaragoza

Navas, A., García-Ruiz, J.M., Machín, J., Lasanta, T., Walling, D., Quine, T. y VALERO, B. (1997). Aspects of soil erosion in dry farming land in two changing environments of the central Ebro valley, Spain. In: Human Impact on Erosion and Sedimentation (Walling, D.E. \& Probst, J.L. Eds.) IAHS Publi. no 245: 13-20.

Navas, A., Valero-Garcés, B.L., Machín, J. y Walling, D. (1998). Los sedimentos del embalse de Joaquín Costa y la historia de su depósito. Limnética 14: 93-112. 
NAVAS, A., VALERO-GARCÉS, B.L. y Machín, J. (2004). An approach to integrated assessement of reservoir siltation: the Joaquín Costa reservoir as case study. Hydrology and Earth System Sciences, 8 (6): 1193-1199.

NAVAS, A., MAchíN, J. y Soto, J. (2005a). Assessing soil erosion in a Pyrenean mountain catchment using GIS and fallout ${ }^{137} \mathrm{Cs}$. Agriculture, Ecosystems \& Environment, 105: 493-506.

Navas, A., Walling, D., Quine, T., Machín, J. y Soto, J. (2005b). Soil redistribution patterns and factors along a transect in central Ebro basin (NE Spain) and its controls. En: Sediment Budgets (Walling, D.E. \& Horowitz, A.J. Eds.). IAHS Publication, 291: 70-77.

PARry, M., PARry, C. y Livermore, M., Eds. (2000). Valoración de los efectos potenciales del Cambio Climático en Europa (Informe ACACIA de la Comisión europea, Resumen y Conclusiones) Universidad de Castilla-La Mancha-Iberdrola, 29 pág., Toledo.

PEÑA, J.L. (1991). El Relieve. La Geología de España. Ed. Síntesis, 166 pp., Madrid.

SERrano, E. (1998). Geomorfología del Alto Gállego (Pirineo aragonés). Institución 'Fernando El Católico', 501 pp., Zaragoza.

SolÉ-SABARIS, L. (1942). La Canal de Berdún, En: Primera Reunión de Estudios Geográficos, 113-160 pp., Madrid.

Soler, M. y Puigdefábregas, C. (1970). Líneas generales de la geología del Alto Aragón Occidental. Pirineos, 96: 5-21.

SunDBORG, A. y RAPP, A. (1986). Erosion and sedimentation by water: problems and prospects. Ambio, 15: 215-225.

Valero-Garcés, B.L., Navas, A. y Machín, J. (1997). Sediment deposition in the Barasona reservoir, (central Pyrenees, Spain): temporal and spatial variability of sediment yield and land use impacts. Implications for watershed erosion. In: Human Impact on Erosion and Sedimentation (Walling, D.E. \& Probst, J.L. Eds.). IAHS Publi. $n^{\circ}$ 245: 241-250.

VAlero-Garcés, B.L., NAVAs, A., Machín, J. y WAlling, D. (1999). Sediment sources and siltation in mountain reservoirs: a case study from the Central Spanish Pyrenees. Geomorphology, 28: 23-41.

Villar, L., Sesé, J.A. y FerrándeZ, J.V. (1997). Atlas de la Flora del Pirineo aragonés I. Instituto de Estudios Altoaragoneses y Consejo de Protección de la Naturaleza de Aragón, Huesca y Zaragoza, 729 pp., Zaragoza

Villar, L., Sesé, J.A. y Ferrández, J.V. (2001). Atlas de la Flora del Pirineo aragonés II, Instituto de Estudios Altoaragoneses y Consejo de Protección de la Naturaleza de Aragón, Huesca y Zaragoza, 790 pp., Zaragoza. 